\title{
AN INTEGER VALUED SU(3) CASSON INVARIANT
}

\author{
Hans U. Boden, Christopher M. Herald, and Paul Kirk
}

ABSTRACT. We define an integer valued invariant of homology 3 -spheres using the methods of $S U(3)$ gauge theory and study its behavior under orientation reversal and connected sum.

\section{Introduction}

Any $S U(n)$ generalization of the Casson invariant for homology 3 -spheres $X$ ought to be defined as a signed count of conjugacy classes of irreducible $S U(n)$ representations of $\pi_{1} X$. The difficulty is that, just as in the $S U(2)$ case, one must perturb the space of representations to make it generic and hence finite, but for $n>2$ the signed count depends on the perturbation.

In [9], Taubes introduced a technique for perturbing the flatness equations and gave a gauge-theoretic interpretation of Casson's $S U(2)$ invariant as a signed count of gauge orbits of perturbed flat $S U(2)$ connections on $X$. In [2], an $S U(3)$ Casson invariant $\lambda_{S U(3)}$ for homology 3-spheres $X$ is defined using the perturbation approach of Taubes. For each generic perturbation $h$, an integer $\lambda_{S U(3)}^{\prime}(X, h)$ is defined as a signed count of gauge orbits of irreducible, $h$ perturbed flat $\mathrm{SU}(3)$ connections,

$$
\lambda_{S U(3)}^{\prime}(X, h)=\sum_{[A] \in \mathcal{M}_{h}^{*}}(-1)^{S F(\theta, A)},
$$

where $\mathcal{M}_{h}^{*}$ is the moduli space of irreducible, $h$-perturbed flat $S U(3)$ connections, $A$ is a representative for the gauge orbit $[A]$, and $S F$ refers to the spectral flow of the odd signature operator $K_{A}$ acting on $s u(3)$-valued forms.

The resulting integer $\lambda_{S U(3)}^{\prime}(X, h)$ is not independent of the choice of the perturbation $h$. To extract a topological invariant, one needs to define a correction term. An analysis of the parametrized moduli space corresponding to a path joining two generic perturbations suggests that the correction term should be a signed sum of the form

$$
\frac{1}{2} \sum_{[A] \in \mathcal{M}_{h}^{r}}(-1)^{S F(\theta, A)} S F_{\mathfrak{h}^{\perp}}\left(A_{0}, A\right)
$$

Received by the editors August 10, 2000.

The authors gratefully acknowledge partial support from National Science Foundation grants (DMS-9971578, DMS-0072564, and DMS-9971020). The second author was also partially supported by a Junior Faculty Research Award from the University of Nevada, Reno. 
where $\mathcal{M}_{h}^{r}$ is the moduli space of reducible, $h$-perturbed flat connections (i.e. with holonomy reducing to $S(U(2) \times U(1)))$ and $A_{0}$ is some fixed reducible connection. Here, the subscript on $S F_{\mathfrak{h} \perp}$ indicates that the operator $K_{A}$ is acting on forms with coefficients in $\mathfrak{h}^{\perp}$, the orthogonal complement in $s u(3)$ of the Lie subalgebra $\mathfrak{h}=s(u(2) \times u(1))$.

In [2], $A_{0}$ was taken to be $\theta$, the trivial connection. Unfortunately, the quantity $S F_{\mathfrak{h} \perp}(\theta, A)$ is not gauge invariant; it depends on the choice of representative $A$ for the gauge equivalence class $[A]$. It was shown there how to restore gauge invariance by restricting to small perturbations and adding the Chern-Simons invariant of a flat connection $\widehat{A}$ near the representative $A$ to obtain the correction term:

$$
\lambda_{S U(3)}^{\prime \prime}(X, h)=\frac{1}{2} \sum_{[A] \in \mathcal{M}_{h}^{r}}(-1)^{S F(\theta, A)}\left(S F_{\mathfrak{h}^{\perp}}(\theta, A)-4 c s(\widehat{A})+2\right) .
$$

Then $\lambda_{S U(3)}(X)=\lambda_{S U(3)}^{\prime}(X, h)+\lambda_{S U(3)}^{\prime \prime}(X, h)$ is independent of the choice of small perturbation $h$. In the recent preprint [7], Cappell, Lee and Miller develop a different technique for correcting the gauge ambiguity in (2).

In this paper, we construct a correction term $\tau^{\prime \prime}(X, h)$ like $(2)$ but without any gauge ambiguity. Setting

$$
\tau(X)=\lambda_{S U(3)}^{\prime}(X, h)+\tau^{\prime \prime}(X, h)
$$

gives an integer valued $S U(3)$ Casson invariant of homology 3 -spheres. Like $\lambda_{S U(3)}$, the invariant $\tau$ enjoys properties (i) - (iii) of the following theorem, which is our main result.

Theorem 4. The quantity $\tau(X)$ is an integer valued invariant of homology 3-spheres. Furthermore,

(i) If $\tau(X) \neq 0$, then there exists an irreducible representation $\rho: \pi_{1} X \rightarrow$ $S U(3)$.

(ii) If $\bar{X}$ equals $X$ with the orientation reversed, then $\tau(X)=\tau(\bar{X})$.

(iii) If $X_{1}$ and $X_{2}$ are homology 3-spheres, then $\tau\left(X_{1} \# X_{2}\right)=\tau\left(X_{1}\right)+\tau\left(X_{2}\right)+4 \lambda_{S U(2)}\left(X_{1}\right) \lambda_{S U(2)}\left(X_{2}\right)$.

The invariant $\tau$ has numerous advantages over $\lambda_{S U(3)}$, and we mention three. First, $\tau(X) \in \mathbb{Z}$. (A priori $\lambda_{S U(3)}(X)$ takes values in $\mathbb{R}$, although the conjectured rationality of the Chern-Simons invariants would imply $\lambda_{S U(3)}(X) \in \mathbb{Q}$.) Secondly, because Casson's invariant is a finite type invariant, one expects the same is true of the generalized Casson invariants. This is not the case for $\lambda_{S U(3)}$; the computations in [4] imply $\lambda_{S U(3)}$ is not a finite type invariant. Nevertheless, those same computations support the conjecture that $\tau$ is related to finite type invariants. Thirdly and most importantly, $\tau$ is easier to compute than $\lambda_{S U(3)}$ and therefore seems more likely to satisfy a surgery formula. 
To illustrate this last point, we suppose that the moduli space $\mathcal{M}$ of unperturbed flat $S U(3)$ connections on $X$ is regular. This is equivalent to the topological assertion

$$
H_{\alpha}^{1}(X ; s u(3))=0 \text { for all representations } \alpha: \pi_{1} X \rightarrow S U(3) .
$$

For example, every Brieskorn sphere of the form $\Sigma(2, p, q)$ satisfies $(*)$. If $X$ satisfies $(*)$, then $\lambda_{S U(3)}(X)$ can be computed directly from $\mathcal{M}$ without resorting to the use of perturbations. I.e. one can take $h=0$. In this case, the correction term $\lambda_{S U(3)}^{\prime \prime}$ is simply a signed sum Atiyah-Patodi-Singer rho invariants, but computing $\lambda_{S U(3)}^{\prime \prime}(X)$ is somewhat involved (cf. the computations in [4]).

By contrast, whenever $(*)$ holds the correction term $\tau^{\prime \prime}$ vanishes. Hence, $\tau(X)=\lambda_{S U(3)}^{\prime}(X, 0)$ whenever $\mathcal{M}$ is regular. This nice property of $\tau$ holds in the more general situation of homology 3 -spheres satisfying

$$
H_{\alpha}^{1}\left(X ; \mathbb{C}^{2}\right)=0 \text { for all representations } \alpha: \pi_{1} X \rightarrow S U(2) .
$$

The condition (**) implies that $\lambda_{S U(3)}^{\prime}(X, h)$ and $\lambda_{S U(3)}^{\prime \prime}(X, h)$ are each independent of $h$ small. In Lemma 7 , we prove that, whenever $(* *)$ holds, $\tau^{\prime \prime}(X, h)=0$ and $\tau(X)=\lambda_{S U(3)}^{\prime}(X, h)$ for $h$ small.

Of course, many homology 3 -spheres fail to satisfy $(* *)$; examples include Brieskorn spheres of the form $\Sigma(p, q, r)$ with $p, q, r>2$. Thus the integer $\lambda_{S U(3)}^{\prime}(X, h)$ will generally depend on the choice of $h$. Nevertheless, $\tau(X)$ agrees with $\lambda_{S U(3)}^{\prime}(X, h)$ whenever the latter is independent of $h$. In this sense, $\tau$ is a topological invariant of homology 3 -spheres which accounts for gauge orbits of reducible connections only when absolutely necessary.

Notation. Throughout this paper, $X$ will be a homology 3 -sphere, i.e. a closed, oriented 3 -manifold satisfying $H_{i}(X ; \mathbb{Z})=H_{i}\left(S^{3} ; \mathbb{Z}\right)$. We denote by $\bar{X}$ the oppositely oriented homology 3 -sphere.

Let $\lambda_{S U(2)}(X)$ be Casson's original invariant, as normalized in [10] (so $\lambda_{S U(2)}(X) \in 2 \mathbb{Z}$ ), and $\lambda_{S U(3)}(X)$ be the invariant defined in [2].

For convenience, we use the notation for differential forms whereby $\Omega^{0+1}=$ $\Omega^{0} \oplus \Omega^{1}$, and similarly for cohomology. Additionally, we use the shorthand $h^{i}=\operatorname{dim} H^{i}$.

Given a path $K_{t}, t \in[0,1]$ of operators with discrete, real spectrum, we denote by $S F\left(K_{t}\right)$ the spectral flow of $K_{t}$ from $t=0$ to $t=1$ using the $(-\epsilon,-\epsilon)$ convention. Thus, $S F\left(K_{t}\right)$ is the oriented intersection number in $[0,1] \times \mathbb{R}$ of the spectrum of $K_{t}$ with the horizontal line segment $(t,-\epsilon), t \in[0,1]$ for all $\epsilon>0$ sufficiently small. With this convention, spectral flow is additive under composition of paths.

\section{Main results}

We begin with a review of 3 -manifold $S U(3)$ gauge theory. Let $X$ be a homology sphere, $P=X \times S U(3)$, and $\theta$ be the trivial (product) connection on $P$, 
with covariant derivative $d$. We denote the space of smooth $S U(3)$ connections on $P$ by

$$
\mathcal{A}=\left\{d+A \mid A \in \Omega^{1}(X ; s u(3))\right\}
$$

and the gauge group of smooth bundle automorphisms by

$$
\mathcal{G} \cong\{g: X \rightarrow S U(3)\} .
$$

This group acts on $\mathcal{A}$ by $d+A \mapsto d+g A g^{-1}+g d g^{-1}$ with quotient $\mathcal{B}=\mathcal{A} / \mathcal{G}$, the space of gauge orbits. We refer to the connection $d+A$ simply as $A$ when no confusion can arise and use $[A]$ to denote the $\mathcal{G}$ orbit of $A \in \mathcal{A}$.

Let $\mathcal{G}_{0}$ be the identity component of $\mathcal{G}$ and set $\widetilde{\mathcal{B}}=\mathcal{A} / \mathcal{G}_{0}$. Given $A \in \mathcal{A}$, we denote its $\mathcal{G}_{0}$ orbit by $\llbracket A \rrbracket$. Since $\mathcal{G}_{0}$ is the kernel of deg: $\mathcal{G} \rightarrow \mathbb{Z}$ (see Prop. 4.2 in [2]), the natural projection $\widetilde{\mathcal{B}} \rightarrow \mathcal{B}$ is a nontrivial connected $\mathbb{Z}$-cover (in fact the universal cover).

The Chern-Simons function $c s: \mathcal{A} \rightarrow \mathbb{R}$ is defined by the formula

$$
\operatorname{cs}(A)=\frac{1}{8 \pi^{2}} \int_{X} \operatorname{tr}\left(A \wedge d A+\frac{2}{3} A \wedge A \wedge A\right) .
$$

Since $c s(g \cdot A)=c s(A)+\operatorname{deg}(g)$, the Chern-Simons function is a well-defined $\mathbb{R}$-valued function on $\widetilde{\mathcal{B}}$, whereas on $\mathcal{B}$ it takes values in $\mathbb{R} / \mathbb{Z}$.

The situation for the spectral flow is similar. Choose a Riemannian metric on $X$. Let $\mathcal{F}$ denote the space of admissible perturbation functions (see [2] for details). To each pair $(A, h) \in \mathcal{A} \times \mathcal{F}$ we associate a self-adjoint, Fredholm operator $K_{A, h}$ on $\Omega^{0+1}(X ; s u(3))$ defined by the formula

$$
K_{A, h}(\xi, a)=\left(d_{A}^{*} a, d_{A} \xi+* d_{A} a-4 \pi \operatorname{Hess} h(A) a\right) .
$$

If $h=0$, then $K_{A, 0}=K_{A}$ is simply the odd signature operator coupled to the connection. If $A$ is flat, then the Hodge and de Rham theorems identify ker $K_{A}$ with the twisted cohomology $H_{\alpha}^{0+1}(X ; s u(3))$, where $\alpha=h o l_{A}: \pi_{1} X \rightarrow S U(3)$ is the holonomy representation of $A$. Hereafter, this cohomology will be denoted by $H_{A}^{0+1}(X ; s u(3))$. More generally, when $A$ is $h$-perturbed flat, we can decompose ker $K_{A, h}$ by degree and write

$$
\operatorname{ker} K_{A, h}=H_{A}^{0}(X ; s u(3)) \oplus H_{A, h}^{1}(X ; s u(3)) .
$$

Note that $H_{A}^{0}(X ; s u(3))=\operatorname{ker}\left(d_{A}: \Omega^{0}(X ; s u(3)) \rightarrow \Omega^{1}(X ; s u(3))\right.$ is independent of $h$ and can be identified with the Lie algebra of the stabilizer of $A$ in $\mathcal{G}$.

Given $\left(A_{t}, h_{t}\right), 0 \leq t \leq 1$, in $\mathcal{A} \times \mathcal{F}$, the spectral flow of the path of self-adjoint operators $K_{A_{t}, h_{t}}, 0 \leq t \leq 1$, is an integer-valued invariant of the homotopy class of the path rel endpoints. Because $\mathcal{A}$ and $\mathcal{F}$ are simply connected, this spectral flow depends only on the endpoints $\left(A_{0}, h_{0}\right)$ and $\left(A_{1}, h_{1}\right)$.

To avoid cumbersome notation, we will adopt the following conventions. If $A_{0}$ and $A_{1}$ are flat connections, then $\operatorname{SF}\left(A_{0}, A_{1}\right)$ will always mean the spectral flow of $K_{A_{t}, 0}$ for $A_{t}, t \in[0,1]$ a path from $A_{0}$ to $A_{1}$. If $A_{0}$ is flat (e.g., $A_{0}$ is the trivial connection $\theta$ ) and $A_{1}$ is $h$-perturbed flat for a fixed perturbation $h$, then $S F\left(A_{0}, A_{1}\right)$ will always denote the spectral flow of $K_{A_{t}, h_{t}}$ for $A_{t}, t \in[0,1]$ a path of connections from $A_{0}$ to $A_{1}$ and $h_{t}$ a path of perturbations from $h_{0}=0$ 
to $h_{1}=h$, i.e. the spectral flow of the path of self-adjoint operators from $K_{A_{0}, 0}$ to $K_{A_{1}, h}$. In all other contexts the choice of the path $h_{t}$ will be specified (or obvious) and $S F\left(A_{0}, A_{1}\right)$ will denote the spectral flow of $K_{A_{t}, h_{t}}$.

Given a connection $A$ and a gauge transformation $g$, the index theorem implies that the spectral flow of $K_{A_{t}, 0}$ along a path $A_{t}$ from $A_{0}=A$ to $A_{1}=g \cdot A$ equals $12 \operatorname{deg} g$ (for a demonstration of this, see [8]). Thus the function $A \mapsto S F(\theta, A)$ on connections descends to a well-defined function $\widetilde{\mathcal{B}}=\widetilde{\mathcal{B}} \times\{0\} \rightarrow \mathbb{Z}$ or to a function $\mathcal{B}=\mathcal{B} \times\{0\} \rightarrow \mathbb{Z}_{12}$.

Let $F_{A}=d A+A \wedge A$ be the curvature of the connection $\mathrm{A}$ and $\mathcal{M}$ the moduli space of flat $S U(3)$ connections

$$
\mathcal{M}=\left\{A \in \mathcal{A} \mid F_{A}=0\right\} / \mathcal{G} .
$$

Note that $\mathcal{M}$ is a compact subset of $\mathcal{B}$ since it is homeomorphic to the space of conjugacy classes of $S U(3)$ representations of $\pi_{1} X$. Its preimage in $\mathcal{A}$ is precisely the set of critical points of the Chern-Simons function. Given an admissible perturbation $h$ (see Definition 2.1 of [2]), a connection $A$ is called $h$-perturbed flat if it is a critical point of the function $c s+h: \mathcal{A} \rightarrow \mathbb{R}$. We denote the moduli space of $h$-perturbed flat connections by $\mathcal{M}_{h}$; it is compact by Lemma 8.3 of [9]. The moduli spaces of $\mathcal{G}_{0}$ orbits of flat connections and of $h$-perturbed flat connections are denoted $\widetilde{\mathcal{M}}$ and $\widetilde{\mathcal{M}}_{h}$. Since $\widetilde{\mathcal{B}} \rightarrow \mathcal{B}$ is a $\mathbb{Z}$-cover, neither $\widetilde{\mathcal{M}}$ nor $\widetilde{\mathcal{M}}_{h}$ is compact.

Let $\Gamma_{A}=\{g \in \mathcal{G} \mid g \cdot A=A\}$ be the isotropy group of $A \in \mathcal{A}$ and define the subsets

$$
\mathcal{A}^{*}=\left\{A \in \mathcal{A} \mid \Gamma_{A} \cong \mathbb{Z}_{3}\right\} \quad \text { and } \quad \mathcal{A}^{r}=\left\{A \in \mathcal{A} \mid \Gamma_{A} \cong U(1)\right\},
$$

of irreducible connections and reducible, nonabelian connections, respectively. Since $X$ is a homology sphere, $\mathcal{M}$ decomposes as the disjoint union

$$
\mathcal{M}=\{[\theta]\} \cup \mathcal{M}^{r} \cup \mathcal{M}^{*} .
$$

We denote by $\mathcal{A}_{S(U(2) \times U(1))}$ the subset of $\mathcal{A}$ consisting of connections $A$ whose holonomy reduces to the standard $S(U(2) \times U(1))$ subgroup. For such connections, $K_{A, h}$ acts diagonally with respect to the splitting

$$
\Omega^{0+1}(X ; s u(3))=\Omega^{0+1}(X ; \mathfrak{h}) \oplus \Omega^{0+1}\left(X ; \mathfrak{h}^{\perp}\right)
$$

associated to the splitting $s u(3)=\mathfrak{h} \oplus \mathfrak{h}^{\perp}$, where $\mathfrak{h}=s(u(2) \times u(1))$ and $\mathfrak{h}^{\perp} \cong$ $\mathbb{C}^{2}$ is its orthogonal complement. For any $A_{0}, A_{1} \in \mathcal{A}^{r}$, choose a path in $\mathcal{A}^{r}$ between them $\left(\mathcal{A}^{r}\right.$ is path connected) and gauge transform so the path lies in $\mathcal{A}_{S(U(2) \times U(1))}$. Then the spectral flow splits according to the decomposition of (4) as

$$
S F\left(A_{0}, A_{1}\right)=S F_{\mathfrak{h}}\left(A_{0}, A_{1}\right)+S F_{\mathfrak{h} \perp}\left(A_{0}, A_{1}\right) .
$$

If, in addition, $A \in \mathcal{A}_{S(U(2) \times U(1))}$ is $h$-perturbed flat, then we have decompositions 


$$
\begin{aligned}
H_{A}^{0}(X ; s u(3)) & =H_{A}^{0}(X ; \mathfrak{h}) \oplus H_{A}^{0}\left(X ; \mathfrak{h}^{\perp}\right), \\
H_{A, h}^{1}(X ; s u(3)) & =H_{A, h}^{1}(X ; \mathfrak{h}) \oplus H_{A, h}^{1}\left(X ; \mathfrak{h}^{\perp}\right) .
\end{aligned}
$$

In this case, it is not hard to show that $H_{A}^{0}(X ; \mathfrak{h}) \cong \mathbb{R}$ and $H_{A}^{0}\left(X ; \mathfrak{h}^{\perp}\right)=0$.

Proposition 1. On any component $\widetilde{C} \subset \widetilde{\mathcal{M}}^{r}$, the function $\widetilde{C} \rightarrow \mathbb{Z}$ defined by $\llbracket A \rrbracket \mapsto S F_{\mathfrak{h} \perp}(\theta, A)$ is bounded above and below.

Proof. Since the Chern-Simons function is constant on components of flat connections and since $c s: \mathcal{B} \rightarrow \mathbb{R} / \mathbb{Z}$ classifies the $\mathbb{Z}$-cover $\widetilde{\mathcal{B}} \rightarrow \mathcal{B}$, it follows that $\widetilde{\mathcal{M}} \rightarrow \mathcal{M}$ is the trivial $\mathbb{Z}$-cover. Thus, every such $\widetilde{C}$ is a homeomorphic copy of a component of $\mathcal{M}^{r}$ and is therefore compact.

Choose $[A] \in \mathcal{M}^{r}$. Then $A$ is nonabelian, and we can assume after gauge transformation that $A \in \mathcal{A}_{S(U(2) \times U(1))}$. The zeroth cohomology $H_{A}^{0}(X ; s u(3))$ then consists of 0 -forms which are constant diagonal matrices of the form

$$
\left(\begin{array}{ccc}
i a & 0 & 0 \\
0 & i a & 0 \\
0 & 0 & -2 i a
\end{array}\right) .
$$

In particular, this implies that $H_{A}^{0}\left(X ; \mathfrak{h}^{\perp}\right)=0$ and $H_{A}^{1}\left(X ; \mathfrak{h}^{\perp}\right)$ is identified with the kernel of $K_{A, h}$ acting on $\mathfrak{h}^{\perp}$-valued forms.

Since the dimension of the kernel of a continuous family of Fredholm operators is upper semicontinuous, $h_{A}^{1}\left(X ; \mathfrak{h}^{\perp}\right)$ is a bounded function on the compact set $\mathcal{M}^{r}$. Since we are using the $(-\epsilon,-\epsilon)$ convention, it follows that every $\llbracket A \rrbracket \in \widetilde{C}$ is contained in a neighborhood $U$ such that

$$
S F_{\mathfrak{h}^{\perp}}(\theta, A)-h_{A}^{1}\left(X ; \mathfrak{h}^{\perp}\right) \leq S F_{\mathfrak{h}^{\perp}}\left(\theta, A^{\prime}\right) \leq S F_{\mathfrak{h}^{\perp}}(\theta, A)
$$

for all $A^{\prime} \in U$. Taking one such neighborhood for each $\llbracket A \rrbracket \in \widetilde{C}$ gives an open covering of $\widetilde{C}$. Using compactness to pass to a finite subcover, we conclude that the function $\llbracket A \rrbracket \mapsto S F_{\mathfrak{h} \perp}(\theta, A)$ is bounded above and below.

For the remainder of this paper, we denote by $C_{1}, \ldots, C_{n}$ the connected components of $\mathcal{M}^{r}$ and by $U_{1}, \ldots, U_{n}$ disjoint open sets of $\mathcal{B}^{r}=\mathcal{A}^{r} / \mathcal{G}$ with $C_{i} \subset U_{i}$ for $i=1, \ldots n$. Since the Chern-Simons function $c s: \mathcal{B}^{r} \rightarrow \mathbb{R} / \mathbb{Z}$ is constant along the components $C_{i}$, we can choose $U_{i}$ small enough so that $c s\left(U_{i}\right)$ is a proper subset of $\mathbb{R} / \mathbb{Z}$. This condition guarantees that the restriction of $p: \widetilde{\mathcal{B}}^{r} \rightarrow \mathcal{B}^{r}$ to $p^{-1}\left(U_{i}\right)$ is the trivial $\mathbb{Z}$-cover.

Proposition 3.7 of [2] insures that for $h \in \mathcal{F}$ sufficiently small, we have $\mathcal{M}_{h}^{r} \subset \bigcup_{i=1}^{n} U_{i}$. If $\left[A_{0}\right],\left[A_{1}\right] \in U_{i}$, there is an unambiguous way to define $\mathfrak{h}^{\perp}$ spectral flow between them by choosing gauge representatives in the same component of $p^{-1}\left(U_{i}\right)$. (Which component of $p^{-1}\left(U_{i}\right)$ they lie in does not matter, since $S F_{\mathfrak{h} \perp}\left(A_{0}, A_{1}\right)=S F_{\mathfrak{h} \perp}\left(g \cdot A_{0}, g \cdot A_{1}\right)$. $)$ In this situation, we will call the representatives $A_{0}, A_{1}$ of $\left[A_{0}\right],\left[A_{1}\right]$ compatible with one another. Any time we 
refer to $\mathfrak{h}^{\perp}$ spectral flow between nontrivial reducible connections, we assume the connections are compatible.

Proposition 1 has the following consequence.

Corollary 2. There exist $\left[\widehat{A}_{i}^{+}\right],\left[\widehat{A}_{i}^{-}\right] \in C_{i}, i=1, \ldots, n$, such that for all $[A] \in$ $C_{i}$ and any mutually compatible representatives $A, \widehat{A}_{i}^{+}, \widehat{A}_{i}^{-}$,

$$
\begin{aligned}
& S F_{\mathfrak{h}^{\perp}}(\theta, A) \leq S F_{\mathfrak{h}^{\perp}}\left(\theta, \widehat{A}_{i}^{+}\right), \\
& S F_{\mathfrak{h}^{\perp}}\left(\theta, \widehat{A}_{i}^{-}\right)-h_{\hat{A}_{i}^{-}}^{1}\left(X ; \mathfrak{h}^{\perp}\right) \leq S F_{\mathfrak{h}^{\perp}}(\theta, A)-h_{A}^{1}\left(X ; \mathfrak{h}^{\perp}\right) .
\end{aligned}
$$

For generic small $h \in \mathcal{F}, \mathcal{M}_{h}$ is regular, i.e. for all $[A] \in \mathcal{M}_{h}$ the condition $H_{A, h}^{1}(X ; s u(3))=0$ holds. (See Section 3 of [2] for details.) Regularity implies that $\mathcal{M}_{h}$ consists of only finitely many points.

Proposition 3. Suppose $h$ is a small, generic perturbation. Define

$$
\begin{aligned}
\tau^{\prime \prime}(X, h)=\frac{1}{4} \sum_{i=1}^{n} \sum_{[A] \in \mathcal{M}_{h}^{r} \cap U_{i}}(-1)^{S F(\theta, A)}\left(S F_{\mathfrak{h}^{\perp}}\left(\widehat{A}_{i}^{+}, A\right)\right. \\
\left.+S F_{\mathfrak{h}^{\perp}}\left(\widehat{A}_{i}^{-}, A\right)+h_{\hat{A}_{i}^{-}}^{1}\left(X ; \mathfrak{h}^{\perp}\right)\right) .
\end{aligned}
$$

Then $\tau^{\prime \prime}(X, h)$ is an integer and depends only on the perturbation $h$ and the manifold $X$; in particular it is independent of the choice of $\widehat{A}_{i}^{ \pm}$.

Proof. We first prove that $\tau^{\prime \prime}(X, h)$ depends only on the perturbation $h$. Since we have already seen that the spectral flow terms are gauge invariant, we just need to show that $\tau^{\prime \prime}(X, h)$ is independent of the choices of $\left[\widehat{A}_{i}^{+}\right],\left[\widehat{A}_{i}^{-}\right] \in C_{i}$ for $i=1, \ldots, n$. Suppose then that $\left[\widehat{B}_{i}^{+}\right],\left[\widehat{B}_{i}^{-}\right] \in C_{i}$ also satisfy Corollary 2. Taking lifts $\widehat{B}_{i}^{+}, \widehat{B}_{i}^{-}$compatible with $\widehat{A}_{i}^{+}, \widehat{A}_{i}^{-}$, it follows from additivity of spectral flow and Corollary 2 that

$$
\begin{aligned}
S F_{\mathfrak{h}^{\perp}}\left(\widehat{A}_{i}^{+}, A\right) & =S F_{\mathfrak{h}^{\perp}}\left(\widehat{B}_{i}^{+}, A\right), \\
S F_{\mathfrak{h}^{\perp}}\left(\widehat{A}_{i}^{-}, A\right)+h_{\hat{A}_{i}^{-}}^{1}\left(X ; \mathfrak{h}^{\perp}\right) & =S F_{\mathfrak{h}^{\perp}}\left(\widehat{B}_{i}^{-}, A\right)+h_{\hat{B}_{i}^{+}}^{1}\left(X ; \mathfrak{h}^{\perp}\right) .
\end{aligned}
$$

for all $A \in \mathcal{A}^{r}$. This shows $\tau^{\prime \prime}(X, h)$ is independent of the choice of $\left[\widehat{A}_{i}^{+}\right],\left[\widehat{A}_{i}^{-}\right] \in$ $C_{i}$ satisfying Corollary 2.

To show $\tau^{\prime \prime}(X, h) \in \mathbb{Z}$, we claim that

$$
S F_{\mathfrak{h}^{\perp}}\left(\widehat{A}_{i}^{-}, A\right)+S F_{\mathfrak{h}^{\perp}}\left(\widehat{A}_{i}^{+}, A\right)+h_{\hat{A}_{i}^{-}}^{1}\left(X ; \mathfrak{h}^{\perp}\right)
$$

is divisible by 4 for all $[A] \in \mathcal{B}^{r}$. Additivity of the spectral flow gives that (6) equals

$$
2 S F_{\mathfrak{h}^{\perp}}\left(\widehat{A}_{i}^{-}, A\right)-S F_{\mathfrak{h}^{\perp}}\left(\widehat{A}_{i}^{-}, \widehat{A}_{i}^{+}\right)+h_{\hat{A}_{i}^{-}}^{1}\left(X ; \mathfrak{h}^{\perp}\right) .
$$

We claim that each of these three terms is divisible by 4 .

Divisibility of the first term follows because the $\Gamma_{A} \cong U(1)$ action on $\Omega^{0+1}\left(X ; \mathfrak{h}^{\perp}\right)$ gives rise to a complex structure with which $K_{A_{t}, h_{t}}$ commutes for 
each t, where $\left(A_{t}, h_{t}\right)$ is a path from $\left(\widehat{A}_{i}^{-}, 0\right)$ to $(A, h)$. This implies $S F_{\mathfrak{h}^{\perp}}\left(\widehat{A}_{i}^{-}, A\right)$ is even.

Divisibility of the second and third terms is a consequence of the following claim.

Claim. If $A_{1}$ and $A_{2}$ are $S U(2) \times\{1\}$ connections, then $S F_{\mathfrak{h} \perp}\left(A_{1}, A_{2}\right)$ and $h_{A_{i}}^{1}\left(X ; \mathfrak{h}^{\perp}\right)$ are divisible by four.

To see this, identify $S U(2)$ with $S p(1)$, the unit quaternions, and $\mathfrak{h}^{\perp} \cong \mathbb{C}^{2}$ with $\mathbb{H}$, the quaternions. The standard representation of $S U(2)$ on $\mathbb{C}^{2}$ can then be viewed as left multiplication in $\mathbb{H}$, and it follows that right multiplication in $\mathbb{H}$ endows each eigenspace of $K_{A}$ with a quaternionic structure. This proves the claim.

The following theorem is our main result.

Theorem 4. Suppose $h$ is a small generic perturbation. Set

$$
\tau(X)=\lambda_{S U(3)}^{\prime}(X, h)+\tau^{\prime \prime}(X, h)
$$

where $\lambda_{S U(3)}^{\prime}(X, h)$ is defined in equation (1) and $\tau^{\prime \prime}(X, h)$ is given in Proposition 3. Then $\tau(X)$ is an integer valued invariant of homology 3-spheres which agrees with $\lambda_{S U(3)}^{\prime}$ on homology 3-spheres satisfying (**). Furthermore

(i) If $\tau(X) \neq 0$, then there exists an irreducible representation $\rho: \pi_{1} X \rightarrow$ $S U(3)$.

(ii) $\tau(X)=\tau(\bar{X})$.

(iii) If $X_{1}$ and $X_{2}$ are homology 3-spheres, then $\tau\left(X_{1} \# X_{2}\right)=\tau\left(X_{1}\right)+\tau\left(X_{2}\right)+4 \lambda_{S U(2)}\left(X_{1}\right) \lambda_{S U(2)}\left(X_{2}\right)$.

\section{Proofs}

Although it is possible to give a direct proof of Theorem 4 based on the arguments of $[2,3]$, it is in fact easier and more informative to study the difference between $\lambda_{S U(3)}$ and $\tau$. This is the approach we take in proving Theorem 4 . The principal result is Lemma 6 , where we identify $\lambda_{S U(3)}-\tau$ in terms of the following more general construction.

Proposition 5. Recall that $C_{1}, \ldots, C_{n}$ are the connected components of $\mathcal{M}^{r}$ with disjoint neighborhoods $U_{1}, \ldots, U_{n}$ in $\mathcal{B}^{r}$. Given any $\alpha_{1}, \ldots, \alpha_{n} \in \mathbb{R}$, the quantity

$$
\sum_{i=1}^{n} \sum_{[A] \in \mathcal{M}_{h}^{r} \cap U_{i}}(-1)^{S F(\theta, A)} \alpha_{i},
$$

is independent of choice of generic small perturbation $h$.

Proof. Notice that if $\alpha_{i}=1$ for all $i$, the quantity (7) equals $\lambda_{S U(2)}(X)$ by [9]. The following argument is a simple generalization of the fact that $\lambda_{S U(2)}$ is independent of perturbation. 

Let

Suppose $\rho=\left\{h_{t}, 0 \leq t \leq 1\right\}$, is a generic 1-parameter family of perturbations.

$$
W_{\rho}=\bigcup_{t \in[0,1]} \mathcal{M}_{h_{t}} \times\{t\}
$$

be the parameterized moduli space. Recall that $W_{\rho}^{r}$ is a smooth 1-manifold. If all the perturbations $h_{t}$ in the path are sufficiently small, then $W_{\rho}^{r} \subset \bigcup_{i=1}^{n} U_{i}$, hence for each $i=1, \ldots, n, W_{\rho}^{r} \cap U_{i}$ gives a 1-dimensional cobordism from $\mathcal{M}_{h_{0}}^{r} \cap U_{i}$ to $\mathcal{M}_{h_{1}}^{r} \cap U_{i}$ with orientations given by the spectral flow. Thus each sum $\sum_{[A] \in \mathcal{M}_{h}^{r} \cap U_{i}}(-1)^{S F(\theta, A)}$ is independent of $h$, which proves the proposition.

The numbers $\alpha_{i}$ we use to analyze the difference $\lambda_{S U(3)}-\tau$ are easiest to describe in terms of the Atiyah-Patodi-Singer rho invariants. Since $X$ is a homology 3 -sphere, every $[A] \in \mathcal{M}^{r}$ can be represented by a flat $S U(2) \times\{1\}$ connection. Given such a connection $A$ on $X$, the rho invariant of $A$ with respect to the regular representation of $S U(2)$ on $\mathbb{C}^{2}$ can be defined by the formula (cf. Theorem 5.7, [4])

$$
\varrho(A)=S F_{\mathbb{C}^{2}}(\theta, A)-4 c s(A)+2-\frac{1}{2} h_{A}^{1}\left(X ; \mathbb{C}^{2}\right) .
$$

Equivalently, we can replace $\mathbb{C}^{2}$ coefficients by the subspace of $s u(3)$ which we have been denoting by $\mathfrak{h}^{\perp}$. The rho invariant $\varrho(A)$ depends only on the gauge orbit $[A]$, not the representative.

For $i=1, \ldots, n$, we define numbers

$$
\begin{aligned}
\alpha_{i}^{+} & =\max _{[A] \in C_{i}}\left\{\varrho(A)+\frac{1}{2} h_{A}^{1}\left(X ; \mathfrak{h}^{\perp}\right)\right\}, \\
\alpha_{i}^{-} & =\min _{[A] \in C_{i}}\left\{\varrho(A)-\frac{1}{2} h_{A}^{1}\left(X ; \mathfrak{h}^{\perp}\right)\right\} .
\end{aligned}
$$

It is also useful to let $C_{0}=\{[\theta]\}$ be the component containing the trivial connection and to set $\alpha_{0}^{+}=0=\alpha_{0}^{-}$.

Remark. For each $i=1, \ldots, n$, the connection $\left[\widehat{A}_{i}^{+}\right]$can be characterized in a gauge invariant way as a global maximum point for the function $C_{i} \rightarrow \mathbb{R}$ defined by $[A] \mapsto \varrho(A)+\frac{1}{2} h_{A}^{1}\left(X ; \mathfrak{h}^{\perp}\right)$. This follows by comparing equation (8) and the inequalities (5) since the Chern-Simons function is constant on path components of flat connections. Similarly, $\left[\widehat{A}_{i}^{-}\right]$is a global minimum for the function $C_{i} \rightarrow \mathbb{R}$ defined by $[A] \mapsto \varrho(A)-\frac{1}{2} h_{A}^{1}\left(X ; \mathfrak{h}^{\perp}\right)$. Combining this observation with equations (8) and (9) shows that

$$
\begin{aligned}
\alpha_{i}^{+} & =S F_{\mathfrak{h}^{\perp}}\left(\theta, \widehat{A}_{i}^{+}\right)-4 \operatorname{cs}\left(\widehat{A}_{i}^{+}\right)+2, \\
\alpha_{i}^{-} & =S F_{\mathfrak{h}^{\perp}}\left(\theta, \widehat{A}_{i}^{-}\right)-4 \operatorname{cs}\left(\widehat{A}_{i}^{-}\right)+2-h_{\hat{A}_{i}^{-}}^{1}\left(X ; \mathfrak{h}^{\perp}\right) .
\end{aligned}
$$

Lemma 6. $\tau(X)$ is a topological invariant of homology 3-spheres. 
Proof. Since the invariance of $\lambda_{S U(3)}$ is proved in [2], we only need to prove that $\lambda_{S U(3)}-\tau$ is independent of all choices made. Now

$$
\lambda_{S U(3)}^{\prime \prime}(X, h)=\frac{1}{2} \sum_{[A] \in \mathcal{M}_{h}^{r}}(-1)^{S F(\theta, A)}\left(S F_{\mathfrak{h} \perp}(\theta, A)-4 \operatorname{cs}(\widehat{A})+2\right),
$$

where $\widehat{A}$ is a reducible flat $S U(3)$ connection close to $A$. Of course $[\widehat{A}] \in C_{i}$ for some $i$, and making compatible choices for $\widehat{A}_{i}^{+}$and $\widehat{A}_{i}^{-}$, we see that

$$
\begin{aligned}
\lambda_{S U(3)}(X)-\tau(X)=\lambda_{S U(3)}^{\prime \prime}(X, h)-\tau^{\prime \prime}(X, h) & \\
= & \frac{1}{4} \sum_{i=1}^{n} \sum_{[A] \in \mathcal{M}_{h}^{r} \cap U_{i}}(-1)^{S F(\theta, A)}\left(2 S F_{\mathfrak{h}^{\perp}}(\theta, A)-8 c s(\widehat{A})+4\right. \\
& \left.\quad-S F_{\mathfrak{h}^{\perp}}\left(\widehat{A}_{i}^{+}, A\right)-S F_{\mathfrak{h}^{\perp}}\left(\widehat{A}_{i}^{-}, A\right)-h_{\hat{A}_{i}^{-}}^{1}\left(X ; \mathfrak{h}^{\perp}\right)\right) \\
= & \frac{1}{4} \sum_{i=1}^{n} \sum_{[A] \in \mathcal{M}_{h}^{r} \cap U_{i}}(-1)^{S F(\theta, A)}\left(S F_{\mathfrak{h}^{\perp}}\left(\theta, \widehat{A}_{i}^{+}\right)-4 c s\left(\widehat{A}_{i}^{+}\right)\right. \\
& \left.\quad+S F_{\mathfrak{h}^{\perp}}\left(\theta, \widehat{A}_{i}^{-}\right)-4 c s\left(\widehat{A}_{i}^{-}\right)+4-h_{\hat{A}_{i}^{-}}^{1}\left(X ; \mathfrak{h}^{\perp}\right)\right) \\
= & \frac{1}{4} \sum_{i=1}^{n} \sum_{[A] \in \mathcal{M}_{h}^{r} \cap U_{i}}(-1)^{S F(\theta, A)}\left(\alpha_{i}^{+}+\alpha_{i}^{-}\right) .
\end{aligned}
$$

The third step follows by additivity of the spectral flow together with the fact that $\operatorname{cs}(\widehat{A})=\operatorname{cs}\left(\widehat{A}_{i}^{+}\right)=\operatorname{cs}\left(\widehat{A}_{i}^{-}\right)$, since the Chern-Simons function is constant along connected components of flat connections. Now letting $\alpha_{i}=\alpha_{i}^{+}+\alpha_{i}^{-}$and applying Proposition 5 completes the proof.

Lemma 7. If $X$ satisfies $(* *)$, then $\tau(X)$ equals $\lambda_{S U(3)}^{\prime}(X, h)$ for any small generic perturbation $h$.

Proof. We show that $\tau^{\prime \prime}(X, h)=0$ for sufficiently small $h$ whenever (**) holds, i.e. whenever $H_{A}^{1}\left(X ; \mathfrak{h}^{\perp}\right)=0$ for all $[A] \in \mathcal{M}^{r}$. This cohomology assumption implies $H_{A, h}^{1}\left(X ; \mathfrak{h}^{\perp}\right)$ also vanishes for every $[A] \in \mathcal{M}_{h}^{r}$ for any small $h$. (Note that the assumption of smallness of $h$ here is stronger than the assumption needed to define $\tau$.) Thus $S F_{\mathfrak{h}^{\perp}}\left(\widehat{A}_{i}^{+}, A\right)=0=S F_{\mathfrak{h}^{\perp}}\left(\widehat{A}_{i}^{-}, A\right)$ and $H_{\hat{A}_{i}^{-}}^{1}\left(X ; \mathfrak{h}^{\perp}\right)=0$ for all $[A] \in \mathcal{M}_{h}^{r} \cap U_{i}$. This shows that each summand in the definition of $\tau^{\prime \prime}(X, h)$ vanishes for $h$ sufficiently small.

Lemma 8. $\tau(\bar{X})=\tau(X)$.

Proof. In [2], it is proved that $\lambda_{S U(3)}(\bar{X})=\lambda_{S U(3)}(X)$. So, the lemma follows once we show that $\lambda_{S U(3)}(X)-\tau(X)$ satisfies the same formula.

Reversing the orientation of $X$ changes the sign of the Chern-Simons function but has no effect on the perturbations. Therefore, there is a natural correspondence between the flat moduli spaces $\mathcal{M}_{h}(X)$ and $\mathcal{M}_{-h}(\bar{X})$. Obviously, if $\mathcal{M}_{h}(X)$ is regular, then so is $\mathcal{M}_{-h}(\bar{X})$. 
The odd signature operator $K_{A, h}^{X}$ acts on $\Omega^{0+1}(X ; s u(3))$ by

$$
K_{A, h}^{X}=\left[\begin{array}{cc}
0 & d_{A}^{*} \\
d_{A} & * d_{A}-4 \pi \operatorname{Hess} h(A)
\end{array}\right] .
$$

Changing the orientation of $X$ changes the sign of the Hodge star operator. Replacing $h$ by $-h$ as well, we see that

$$
K_{A,-h}^{\bar{X}}=\left[\begin{array}{cc}
0 & d_{A}^{*} \\
d_{A} & -* d_{A}+4 \pi \operatorname{Hess} h(A)
\end{array}\right] .
$$

Hence, if $K_{A, h}^{X}(\xi, \eta)=\lambda(\xi, \eta)$, then $K_{A,-h}^{\bar{X}}(-\xi, \eta)=-\lambda(-\xi, \eta)$. Thus switching orientations and replacing $h$ by $-h$ reflects the spectrum through zero. The following formula is a consequence of the $(-\epsilon,-\epsilon)$ convention:

$$
S F_{\bar{X}}\left(K_{A_{t},-h_{t}}\right)=-S F_{X}\left(K_{A_{t}, h_{t}}\right)+\operatorname{dim} \operatorname{ker} K_{A_{1}, h_{1}}-\operatorname{dim} \operatorname{ker} K_{A_{0}, h_{0}} .
$$

Now suppose $h$ is a small perturbation and $\mathcal{M}_{h}(X)$ is regular. If $[A] \in \mathcal{M}_{h}^{r}$ then $H_{A}^{0}(X ; s u(3)) \cong \mathbb{R}$ and $H_{A, h}^{1}(X ; s u(3))=0$ and so

$$
S F_{\bar{X}}(\theta, A)=-S F_{X}(\theta, A)+1-8 .
$$

In this formula, on the left the spectral flow is taken from $(\theta, 0)$ to $(A,-h)$, and on the right it is from $(\theta, 0)$ to $(A, h)$.

Further, if $\widehat{A}$ is flat and reducible, then it is a simple exercise to prove $\varrho_{\bar{X}}(\widehat{A})=$ $-\varrho_{X}(\widehat{A})$. Equation $(9)$ then implies that

$$
\begin{aligned}
\alpha_{i}^{+}(\bar{X}) & =\max _{[\hat{A}] \in C_{i}}\left\{\varrho_{\bar{X}}(\widehat{A})+\frac{1}{2} h_{\hat{A}}^{1}\left(X ; \mathfrak{h}^{\perp}\right)\right\} \\
& =\max _{[\hat{A}] \in C_{i}}\left\{-\varrho_{X}(\widehat{A})+\frac{1}{2} h_{\hat{A}}^{1}\left(X ; \mathfrak{h}^{\perp}\right)\right\} \\
& =-\min _{[\hat{A}] \in C_{i}}\left\{\varrho_{X}(\widehat{A})-\frac{1}{2} h_{\hat{A}}^{1}\left(X ; \mathfrak{h}^{\perp}\right)\right\}=-\alpha_{i}^{-}(X) .
\end{aligned}
$$

Similarly $\alpha_{i}^{-}(\bar{X})=-\alpha_{i}^{+}(X)$. Thus

$$
\begin{aligned}
\lambda_{S U(3)}(\bar{X})-\tau(\bar{X}) & =\frac{1}{4} \sum_{i=1}^{n} \sum_{[A] \in \mathcal{M}_{-h}^{r}(\bar{X}) \cap U_{i}}(-1)^{S F_{\bar{X}}(\theta, A)}\left(\alpha_{i}^{+}(\bar{X})+\alpha_{i}^{-}(\bar{X})\right) \\
& =\frac{1}{4} \sum_{i=1}^{n} \sum_{[A] \in \mathcal{M}_{h}^{r}(X) \cap U_{i}}(-1)^{S F_{X}(\theta, A)+1}\left(-\alpha_{i}^{-}(X)-\alpha_{i}^{+}(X)\right) \\
& =\lambda_{S U(3)}(X)-\tau(X) .
\end{aligned}
$$

Lemma 9. If $X_{1}$ and $X_{2}$ are homology 3-spheres, then $\tau\left(X_{1} \# X_{2}\right)=\tau\left(X_{1}\right)+\tau\left(X_{2}\right)+4 \lambda_{S U(2)}\left(X_{1}\right) \lambda_{S U(2)}\left(X_{2}\right)$. 
Proof. Since $\lambda_{S U(3)}$ was shown to satisfy a similar formula in [3], it suffices to show additivity of $\lambda_{S U(3)}-\tau$ under connected sum. We first claim that the numbers $\alpha_{i}^{+}$and $\alpha_{i}^{-}$are additive under connected sum.

To make this precise, we need to set up the notation. Set $X=X_{1} \# X_{2}$. Then every connection $A$ on $X$ is of the form $A=A_{1} \#_{\sigma} A_{2}$, where $A_{i}$ is a connection on $X_{i}$ and $\sigma$ is a gluing parameter. Furthermore, if $A$ is reducible and flat, then so are $A_{1}$ and $A_{2}$.

For $k=1,2$, let $C_{0}\left(X_{k}\right), \ldots, C_{n_{k}}\left(X_{k}\right)$ be the components of $\mathcal{M}^{r}\left(X_{k}\right)$, where $C_{0}\left(X_{k}\right)=\left\{\left[\theta_{k}\right]\right\}$ is the component containing the trivial connection. The components of $\mathcal{M}^{r}(X)$ are then given by the sets

$$
C_{i, j}(X)=\left\{\left[A=A_{1} \#_{\sigma} A_{2}\right] \mid A_{1} \in C_{i}\left(X_{1}\right) \text { and } A_{2} \in C_{j}\left(X_{2}\right)\right\} .
$$

for $0 \leq i \leq n_{1}$ and $0 \leq j \leq n_{2}$. Note that $C_{0,0}(X)$ is now the component containing the trivial connection. For each $i, j$, we also choose an open set $U_{i, j}(X) \subset \mathcal{B}^{r}(X)$ containing $C_{i, j}(X)$ so the collection $\left\{U_{i, j}(X) \mid 0 \leq i \leq n_{1}, 0 \leq\right.$ $\left.j \leq n_{2}\right\}$ is disjoint.

For $k=1,2$ and $i=1, \ldots, n_{k}$, let $\alpha_{i}^{+}\left(X_{k}\right)$ and $\alpha_{i}^{-}\left(X_{k}\right)$ be the quantities defined by equation (9). Writing $\alpha_{i, j}^{+}(X)$ and $\alpha_{i, j}^{-}(X)$ for same numbers defined with respect to the components $C_{i, j}(X)$ for $X=X_{1} \# X_{2}$, we claim that

$$
\begin{aligned}
& \alpha_{i, j}^{+}(X)=\alpha_{i}^{+}\left(X_{1}\right)+\alpha_{j}^{+}\left(X_{2}\right), \\
& \alpha_{i, j}^{-}(X)=\alpha_{i}^{-}\left(X_{1}\right)+\alpha_{j}^{-}\left(X_{2}\right) .
\end{aligned}
$$

To see this, suppose $A=A_{1} \#_{\sigma} A_{2}$ is a reducible flat connection. Adding a 1-handle to $\left(X_{1} \coprod X_{2}\right) \times[0,1]$ gives a flat cobordism from $\left(X_{1}, A_{1}\right) \amalg\left(X_{2}, A_{2}\right)$ to $\left(X_{1} \# X_{2}, A\right)$. Since this cobordism has no 2-handles, its signature and twisted signature vanish, and so the Atiyah-Patodi-Singer index theorem implies that

$$
\varrho_{X_{1} \# X_{2}}(A)=\varrho_{X_{1}}\left(A_{1}\right)+\varrho_{X_{2}}\left(A_{2}\right) .
$$

In addition, the Mayer-Vietoris principle implies

$$
H_{A}^{1}\left(X_{1} \# X_{2} ; \mathfrak{h}^{\perp}\right)=H_{A_{1}}^{1}\left(X_{1} ; \mathfrak{h}^{\perp}\right) \oplus H_{A_{2}}^{1}\left(X_{2} ; \mathfrak{h}^{\perp}\right) .
$$

Equations (10) now follow by applying (11) and (12) above to the definition (9).

Suppose $h_{k}$ is a small admissible perturbation on $X_{k}$ for $k=1,2$, so that $\mathcal{M}_{h_{k}}\left(X_{k}\right)$ is regular. Viewing $h_{1}$ and $h_{2}$ as perturbations on $X=X_{1} \# X_{2}$, set $h_{0}=h_{1}+h_{2}$ and assume the perturbations are chosen small enough so that $\mathcal{M}_{h_{0}}^{r}(X) \subset \bigcup_{i, j} U_{i, j}(X)$.

Given $[A] \in \mathcal{M}_{h_{0}}^{r}(X)$, we can write $A=A_{1} \#_{\sigma} A_{2}$ where $A_{1}$ is an $h_{1}$-perturbed flat reducible connection on $X_{1}$ and $A_{2}$ is an $h_{2}$-perturbed flat reducible connection on $X_{2}$. Then $\mathcal{M}_{h_{0}}^{r}(X)$ consists of two types of components [3]:

(i) $S O(3)$ components of the form $C=\left\{\left[A_{1} \#_{\sigma} A_{2}\right]\right\}$, where $\left[A_{k}\right] \in \mathcal{M}_{h_{k}}^{r}\left(X_{k}\right)$ for $k=1,2$ and $\sigma$ is a gluing parameter with $A_{1} \#_{\sigma} A_{2}$ reducible.

(ii) Point components of the form $C=\left\{\left[\theta_{1} \# A_{2}\right]\right\}$ or $\left\{\left[A_{1} \# \theta_{2}\right]\right\}$, where $\theta_{k}$ is the trivial $S U(3)$ connection over $X_{k}$ and $\left[A_{k}\right] \in \mathcal{M}_{h_{k}}^{r}\left(X_{k}\right)$ for $k=1,2$. 
Note that the intersection $\mathcal{M}_{h_{0}}^{r}(X) \cap U_{i, j}(X)$ consists entirely of components of type (i) unless $i=0$ or $j=0$, in which case it consists of point components.

We first argue that components of type (i) do not contribute to $\lambda_{S U(3)}(X)-$ $\tau(X)$. To see this, suppose $C$ is a component of type (i). Then $C \subset U_{i, j}(X)$ for fixed $i, j>0$. Let $h=h_{0}+t g$ be a perturbation so that the restriction of $g$ to $C$ is Morse. (The existence of such functions is shown in [3].) Then, for small $t$, the contribution of $C$ to $\lambda_{S U(3)}(X)-\tau(X)$ is given by

$$
\left(\alpha_{i, j}^{+}(X)+\alpha_{i, j}^{-}(X)\right) \sum_{p \in \operatorname{Crit}\left(\left.g\right|_{C}\right)}(-1)^{\operatorname{ind}_{p}\left(\left.g\right|_{C}\right)},
$$

which vanishes since the sum evaluates to the Euler characteristic $\chi(C)$ and $C \cong S O(3)$. (This is similar to the proof of Proposition 8 in [3].)

Thus, dropping all the $(i, j)$ terms with $i, j>0$ from the following sum and applying equation (10) to the remaining terms, we conclude that

$$
\begin{aligned}
\lambda_{s u(3)}(X)-\tau(X) & =\frac{1}{4} \sum_{i, j} \sum_{[A] \in \mathcal{M}_{h}^{r}(X) \cap U_{i, j}(X)}(-1)^{S F_{X}(\theta, A)}\left(\alpha_{i, j}^{+}(X)+\alpha_{i, j}^{-}(X)\right) \\
& =\frac{1}{4} \sum_{i=1}^{n_{1}} \sum_{\left[A_{1}\right] \in \mathcal{M}_{h_{1}}^{r}\left(X_{1}\right) \cap U_{i}\left(X_{1}\right)}(-1)^{S F_{X_{1}}\left(\theta_{1}, A_{1}\right)}\left(\alpha_{i}^{+}\left(X_{1}\right)+\alpha_{i}^{-}\left(X_{1}\right)\right) \\
& +\frac{1}{4} \sum_{j=1}^{n_{2}} \sum_{\left[A_{2}\right] \in \mathcal{M}_{h_{2}}^{r}\left(X_{2}\right) \cap U_{j}\left(X_{2}\right)}(-1)^{S F_{X_{2}}\left(\theta_{2}, A_{2}\right)}\left(\alpha_{j}^{+}\left(X_{2}\right)+\alpha_{j}^{-}\left(X_{2}\right)\right) \\
& =\lambda_{S U(3)}\left(X_{1}\right)-\tau\left(X_{1}\right)+\lambda_{S U(3)}\left(X_{2}\right)-\tau\left(X_{2}\right) .
\end{aligned}
$$

Since $\lambda_{S U(2)}(X) \in 2 \mathbb{Z}$, Lemma 9 has the following corollary.

Corollary 10. The mod 16 reduction of $\tau$ is additive with respect to connected sum of homology 3-spheres.

Proof of Theorem 4. Lemmas 6 and 7 show that $\tau(X)$ determines an integer valued invariant of homology spheres $X$ which agrees with $\lambda_{S U(3)}^{\prime}(X)$ for homology 3 -spheres satisfying $(* *)$. Part (i) of Theorem 4 follows since $\tau(X) \neq 0$ implies the existence of an irreducible $S U(2)$ or $S U(3)$ representation of $\pi_{1} X$, but any irreducible $S U(2)$ representation produces an irreducible $S U(3)$ representation via mapping $S U(2) \rightarrow S O(3) \hookrightarrow S U(3)$. Part (ii) follows from Lemma 8 and part (iii) from Lemma 9.

Below are some computations of $\tau$ from [1]. For these examples, observe that $\tau(X)$ is divisible by 2 .

The INVARIANT $\tau$ FOR BRIESKORN SPheres $\Sigma(2, p, q)$ 


\begin{tabular}{|c|c|}
\hline Brieskorn sphere & The invariant $\tau(X)$ \\
\hline \hline$\Sigma(2,3,6 k \pm 1)$ & $3 k^{2} \pm k$ \\
\hline$\Sigma(2,5,10 k \pm 1)$ & $33 k^{2} \pm 9 k$ \\
\hline$\Sigma(2,5,10 k \pm 3)$ & $33 k^{2} \pm 19 k+2$ \\
\hline$\Sigma(2,7,14 k \pm 1)$ & $138 k^{2} \pm 26 k$ \\
\hline$\Sigma(2,7,14 k \pm 3)$ & $138 k^{2} \pm 62 k+4$ \\
\hline$\Sigma(2,7,14 k \pm 5)$ & $138 k^{2} \pm 102 k+16$ \\
\hline$\Sigma(2,9,18 k \pm 1)$ & $390 k^{2} \pm 58 k$ \\
\hline$\Sigma(2,9,18 k \pm 5)$ & $390 k^{2} \pm 210 k+24$ \\
\hline$\Sigma(2,9,18 k \pm 7)$ & $390 k^{2} \pm 298 k+52$ \\
\hline
\end{tabular}

Concluding remarks and open questions. To better understand the relationship between $\lambda_{S U(3)}$ and $\tau$, it is helpful to compare them to the $S U(2)$ invariants $\lambda_{W}$ and $\lambda_{B N}$ of rational homology spheres defined by Kevin Walker [10] and by Boyer and Nicas [5], respectively. Under suitable hypotheses, the difference $\lambda_{W}-\lambda_{B N}$ can be expressed as a sum of Atiyah-Patodi-Singer rho invariants of $U(1)$ representations [6]. A similar statement is true of $\lambda_{S U(3)}-\tau$, and so it is natural to ask whether $\tau$ is the $S U(3)$ analog of the Boyer-Nicas invariant. The answer is no, and we now explain why not.

The Boyer-Nicas approach works more generally for arbitrary compact Lie groups $G$. Their idea is to define a Casson-like invariant by incorporating only compact components of the variety of irreducible representations $\rho: \pi_{1} X \rightarrow$ $G$. For that reason, no correction term is required. In the $S U(3)$ case, their approach would yield an integer valued invariant that presumably agrees with $\tau$ on homology 3 -spheres satisfying $(* *)$.

However, $\tau$ and the $S U(3)$ Boyer-Nicas invariant are not identical; $\tau$ involves a correction term whereas the Boyer-Nicas invariant does not. In truth, $\tau$ incorporates contributions from the reducible components in only a very limited way. The proof of Lemma 7 shows that only those components of $\mathcal{M}^{r}$ which contain gauge orbits $[A]$ with $H_{A}^{1}\left(X ; \mathfrak{h}^{\perp}\right) \neq 0$ contribute to the correction term. These are precisely the components which, from first order considerations, may contain limit points of the irreducible stratum. The corresponding components of the irreducible stratum would therefore be excluded in the Boyer-Nicas approach on the grounds that they are not compact. This illustrates the fundamental difference between $\tau$ and the $S U(3)$ Boyer-Nicas invariant.

Finally, one can ask (as the referee did) what the $S U(2)$ analogue for $\tau$ is for rational homology spheres. In this setting, the reducible (i.e. abelian) stratum of the flat moduli space is already a finite set, so there is a unique choice of basepoint for each component of abelians. The $S U(2)$ analogue of $\tau^{\prime \prime}$ is then a sum of $\mathbb{C}$ spectral flows from the basepoints to the nearby perturbed flat abelian connections. One must decide how to count the zero modes at the basepoints, or, equivalently, which convention to use in computing the $\mathbb{C}$ spectral 
flow. Each of the standard conventions gives a different invariant; one pushes the zero eigenvalues up, the other down. These two invariants should be the gauge theory analogues of the invariants $\lambda_{S U(2)}^{+}$and $\lambda_{S U(2)}^{-}$of [6]. This problem will be addressed in a later paper.

We conclude this paper with five open problems.

1. Is $\tau(X)$ divisible by 2 for all homology 3 -spheres? If not, is $\tau \bmod 2$ a homology cobordism invariant?

2. Establish a relationship between $\tau$ and the finite type invariants.

3. Find a Dehn surgery formula for $\tau$.

4. Compute $\tau$ for homology spheres that do not satisfy (**), e.g., Brieskorn spheres $\Sigma(p, q, r)$ with $p, q, r>2$.

5. Develop an $S U(3)$ Floer theory and relate its Euler characteristic to $\tau$.

\section{References}

[1] H. U. Boden, Unitary representations of Brieskorn spheres, Duke Math. J. 75 (1994), 193-220.

[2] H. U. Boden and C. M. Herald, The SU(3) Casson invariant for integral homology 3spheres, J. Differential Geom. 50 (1998), 147-206.

[3] H. U. Boden and C. M. Herald, A connected sum formula for the SU(3) Casson invariant, J. Differential Geom. 53 (1999), 443-464.

[4] H. U. Boden, C. M. Herald, P. A. Kirk, and E. P. Klassen, Gauge theoretic invariants of Dehn surgeries on knots, Geom. Topol. 5 (2001), 143-226.

[5] S. Boyer and A. Nicas, Varieties of group representations and Casson's invariant for rational homology 3-spheres, Trans. Amer. Math. Soc. 322 (1990), 507-522.

[6] S. E. Cappell, R. Lee and E. Y. Miller, Self-adjoint elliptic operators and manifold decompositions. III. Determinant line bundles and Lagrangian intersection, Comm. Pure Appl. Math. 52 (1999), 543-611.

[7] S. E. Cappell, R. Lee and E. Y. Miller, A perturbative SU(3) Casson invariant, to appear in Commentarii Mathematici Helvetici.

[8] P. Kirk, E. Klassen and D. Ruberman, Splitting the spectral flow and the Alexander matrix, Comment. Math. Helv. 69 (1994), 375-416.

[9] C. H. Taubes, Casson's invariant and gauge theory, J. Differential Geom. 31 (1990), $547-599$.

[10] K. Walker, An extension of Casson's invariant, Annals of Mathematics Studies, 126. Princeton University Press, 1992.

Department of Mathematics and Statistics, McMaster University, Hamilton, ON L8S 4K1 CANADA.

E-mail address: boden@mcmaster.ca

Department of Mathematics, University of Nevada, Reno NV 89557.

E-mail address: herald@unr.edu

Department of Mathematics, Indiana University, Bloomington, in 47405.

E-mail address: pkirk@indiana.edu 